\title{
Type and Portions of Peripheral Blood T Lymphocytes in Oral Lichen Planus
}

\author{
Seyed Javad Kia ${ }^{1}$; Arash Mansourian ${ }^{2, *}$; Masoumeh Nikkhah ${ }^{3}$; Nariman Nikparto ${ }^{4}$; \\ Najmeh Shanbezadeh ${ }^{3}$, \\ ${ }^{1}$ Department of Oral Medicine, Guilan University of Medical Sciences, Rasht, IR Iran \\ 2 Department of Oral Medicine, Gehran University of Medical Sciences, Tehran, IR I \\ 3 Dentist, Private Practice, Tehran, IR Iran \\ ${ }^{4}$ Students' Scientific Research Center, Tehran University of Medical Sciences, Tehran, IR Iran \\ ${ }^{*}$ Corresponding author: Arash Mansourian, Department of Oral Medicine, Tehran University of Medical Sciences, Tehran, IR Iran. Tel: +98-9123344090, Fax: + 98-2188497416, E-mail: \\ Amansourian@tums.ac.ir
}

Received: March 2, 2013; Revised: April 27, 2013; Accepted: June 1, 2013

\begin{abstract}
Background: Lichen planus is a disease with unknown etiology that affects the skin and the mucous membranes. Immune dysregulation in the pathogenesis of oral lichen planus (OLP) is well-known phenomenon.

Objectives: In this study, we compared the levels of the peripheral blood T lymphocytes between patients with OLP and control group. Patients and Methods: In this study, 32 and 16 patients respectively with and without OLP were recruited. Five milliliters of the participants' peripheral venous blood was drew in an EDTA-containing test tube and the levels of CD3+, CD4+, and CD8+ cells, CD4+/CD8+ and CD4+| CD3+ ratio were measured by means of two-color flow cytometry. The data were analyzed in SPPS v.19 by employing Mann-Whitney U test. Results: There were no significant difference among the levels of CD3+, CD4+, and CD8+ lymphocytes and the ratio of CD4+/CD8+ and $\mathrm{CD} 4+/ \mathrm{CD} 3+$ lymphocytes between patients and control group; however, there was a significant difference between male and female patients with respect to the levels of CD3+ and CD4+ lymphocytes and the ratio of CD4+/CD8+ and CD4+/CD3+ lymphocytes.

Conclusions: Our results confirm that only local immune mechanism known as skin-associated lymphoid tissue, not a systemic immunologic disorder, was involved in the OLP.
\end{abstract}

Keywords:Lichen Planus; T-Lymphocytes; Peripheral Blood

\section{Background}

Lichen planus is a chronic inflammatory disease that affects the skin and the mucus membrane. Oral lichen planus (OLP) presents frequently in the fourth decade of life. It is seen clinically as reticular, papular, plaque-like, erosive, atrophic, or bullous types. The buccal mucosa, tongue, and the gingiva are commonly involved in OLP although other sites may be rarely affected. Oral mucosal lesions present alone or with concomitant skin lesions (1). It occurs in $0.1 \%$ to $4 \%$ of the general population, most often in premenopausal women (2). The possible involved factors in the pathogenesis of OLP includes genetic background, immune system defects, stress, diabetes, hypertension, malignancies, bowel disease, chronic liver disease, and viral hepatitis (3). Findings of the immunofluorescent examination of OLP is not specific because cases such as systemic lupus erythematosus and erythema multiform have the similar findings (4). A large body of evidence supports a role of immune dysregulation in the pathogenesis of OLP (5) and its histology characteristics confirm this theory (6). The CD4+ and CD8+ T cells, mast cells, chemokines, antigens, and keratinocytolysis are involved in the immunopathogenesis of OLP (5). By considering the high prevalence of OLP and corticosteroids side effects, finding the main reason and pathogenesis of OLP is an important step in searching for an effective treatment of lichen planus. In a case control study performed by Yamamoto et al. in 1990, cellular immunosuppression in OLP in 20 patients with OLP and 20 healthy individuals was assessed. They found no significant difference in the mean $\mathrm{CD} 3+, \mathrm{CD} 4+$, and $\mathrm{CD} 8+\mathrm{T}$ cell counts and the ratio of CD4+ to CD8+ (7). In 1996, Carrozzo et al. performed a quantitative analysis of peripheral blood lymphocytes in 25 patients with atrophic-erosive OLP, 28 patients with reticular-plaque-like OLP, and 21 healthy individuals. In this study, CD4+ subsets decreased significantly in patients with reticular-plaque-like lesions and CD8+ cell populations were significantly higher than controls and consequently, the ratio of CD4+ to CD8+ cells was significantly lower in patients with reticular-plaque-like OLP than in controls (8). In a study by Charazinska et al. in 2008, no 
Kia SJ et al.

significant difference was found in CD3+ and CD8+ cells populations between patients and controls; however, T helper naive cells decreased significantly and $\mathrm{T}$ helper memory cells increased significantly in OLP patients in comparison to the control group (9).

\section{Objectives}

The objective of our study was to compare of the peripheral blood T-lymphocytes levels between patients with OLP and control group.

\section{Patients and Methods}

In this case-control study, 32 patients (23 females and nine males; mean age, $49.97 \pm 8.50$ years) with reticular, atrophic, erosive, bullous, and plaque-like lesions of OLP who were referred to Oral Disease Department of Dental Faculty of Guilan University of Medical Sciences in 20102011 were recruited. The diagnosis of OLP was made clinically on the basis of typical feathers. Patients who took local or systemic drugs within the preceding month, those with positive history for psychiatric or mental disorders, and woman during pregnancy or lactation were excluded. The control group consisted of 16 volunteers (11 women and five men; mean age, $41.38 \pm 7.14$ years) recruited from family members and friends of the clinic staff. After providing a complete information about the study, written informed consent confirmed by Guilan University of Medical Sciences Research Deputy was obtained. The study was approved by the Local Ethical Committee. Five milliliters of antecubital vain blood was drawn into an EDTA-containing test tube. In all of the participants, blood samples were obtained in the morning. The complete blood count was assessed. Then the blood T-cell levels were measured by means of two-color flow cytometry using Partec PAS (Partec, Munster, Germany). Conjugated antibodies with fluorescent material were added to the peripheral blood and incubated for 30 minutes in $4^{\circ} \mathrm{C}$. Then blood cells were incubated with a lysing agent (UtiLyse solution number 1 and 2, DAKO Corporation, Denmark) to remove erythrocytes. In this method, blood cells were not washed because Uti-Lyse is such solution that the lysing of erythrocytes is done slowly and erythrocytes deposits do not interfere with the results of study. Lymphocyte phenotype analysis was performed using monoclonal antibodies (DAKO, Denmark) to distinguish cells expressing CD3+, CD4+, and CD8+ antigens. Cells passed the laser beam and scattered the laser light. These scatter patterns and fluorescence signals were detected by the cytometry. The cells which were positive for tested antigens (CD4+, CD3+ and CD8+) were included in this study. The obtained results were analyzed by SPSS v.19 (SPSS Inc., Chicago, IL, USA) through Mann-Whitney U test.

\section{Results}

The statistical analysis of the results showed that the mean percentage of $\mathrm{CD} 3+$ and CD4+ T cells was slightly lower in patients with in comparison to the controls group; however, this reduction was not statistically significant $(\mathrm{P}>0.05)$. Moreover, the mean percentage of $\mathrm{CD} 8+\mathrm{T}$ cells was slightly higher in case group in contrast to controls but it was not significant $(\mathrm{P}>0.05)$. The $\mathrm{CD} 4+\mid$ $\mathrm{CD} 8+$ and $\mathrm{CD} 4+/ \mathrm{CD} 3+$ ratios were also lower in case group in comparison to the control group but it was not significant $(\mathrm{P}>0.05)$. In addition, the results of the comparison of the T-cell subsets levels between males and females in the patients group showed statistically significant difference in the level of CD4+ and CD3+ as well as the proportion of $\mathrm{CD} 4+/ \mathrm{CD} 8+$ and $\mathrm{CD} 4+/ \mathrm{CD} 3+\mathrm{T}$ cells $(\mathrm{P}<0.05)$. The mean percentage of $\mathrm{CD} 4+$ and $\mathrm{CD} 3+\mathrm{T}$ cells as well as the $\mathrm{CD} 4+/ \mathrm{CD} 8+$ and $\mathrm{CD} 4+/ \mathrm{CD} 3+$ ratios were higher in females; however, no significant difference was shown between the males and females in the level of CD8+ T cells (Tables 1 and 2).

Table 1. T-Lymphocyte Subsets in Patients With Oral Lichen Planus and Healthy Controls ${ }^{a, b}$

\begin{tabular}{|c|c|c|c|c|c|}
\hline & CD4+ & CD8+ & CD3+ & CD4+/CD8+ Ratio & CD4+/CD3+ Ratio \\
\hline $\operatorname{OLP}(n=32)$ & $39.74 \pm 6.09$ & $24.04 \pm 4.79$ & $65.39 \pm 5.08$ & $1.73 \pm 0.50$ & $0.60 \pm 0.07$ \\
\hline $\operatorname{Control}(n=16)$ & $41.84 \pm 3.61$ & $23.22 \pm 3.07$ & $66.25 \pm 1.90$ & $1.84 \pm 0.42$ & $0.62 \pm 0.06$ \\
\hline PValue & 0.32 & 0.69 & 0.25 & 0.47 & 0.39 \\
\hline
\end{tabular}

a Abbreviation: OLP, oral lichen planus.

$\mathrm{b}$ Data are presented as Mean $\pm S \mathrm{D}$

Table 2. Comparing T-Lymphocyte Subsets between Male and Female Patients with Oral Lichen Planus ${ }^{\text {a }}$

\begin{tabular}{|c|c|c|c|c|c|}
\hline & CD4+ & CD8+ & CD3+ & CD4+/CD8+ Ratio & $\mathrm{CD} 4+/ \mathrm{CD} 3+$ Ratio \\
\hline Male $(\mathbf{n}=9)$ & 9.33 & 17.22 & 11.11 & 10.72 & 10.33 \\
\hline Female $(n=23)$ & 19.3 & 16.22 & 18.61 & 18.76 & 18.91 \\
\hline PValue & 0.007 & 0.785 & 0.042 & 0.029 & 0.02 \\
\hline
\end{tabular}

a Data are presented as mean. 


\section{Discussion}

OLP is a very common oral dermatosis and one of the most frequent mucosal ulcers encountered by dental practitioners. A proper understanding of the pathogenesis of the disease is important for providing the right treatment. The results of this study confirmed the study of Yamamoto et al. (7). They showed no significant difference between the patients and the control group in the level of CD3+, CD4+, CD8+ and CD4+/CD8+. Moreover, the results of our study confirmed the study of Lin et al. (10), Walton et al. (11), Krasowska et al. (12) and Charazinska et al. (9). On the other hand, Carrozzo et al. (8) showed the decreased level of CD4+ and CD4+/CD8+ and increased level of CD8+ in patients; however, they used different statistical tests (t-test, Mann-Whitney U test, and Kruskal-Wallis test). Moreover, they showed these results in patients with reticular-plaque-like lesions in comparison to healthy individuals as control group. alFouzan et al. (13) found that the proportion of both total T cells (CD3+ T cells) and T helper cells (CD4+ T cells) were significantly lower in patients with lichen planus than in healthy controls; however, to properly interpret these authors' findings, it should be remembered that only $30 \%$ of their patients showed oral as well as cutaneous lesions.

According to Eversole (14), the decreased lymphocyte count in the peripheral blood is mainly related to the migration of these cells from the blood vessels and infiltration of the basal skin/mucous membrane layer. Moreover, as the disease progresses and the clinical changes intensify and evolve into erosions, cytotoxic/suppressor cells predominate in the infiltration (15). The very same changes happens in peripheral blood cell counts when the atrophic-erosive lesions are present (16). By considering that about one-third of our patients at the time of sampling had no erosion or pickling in their oral mucosa, observing no significant differences in the level of the blood lymphocytes can be justified.

After treating OLP patients with traditional Chinese medicine, Pang et al. (17) showed a significant increase in the level of CD3+ and CD4+ T cells as well as CD3+/CD4+ ratio. Kia et al. (18) used rotavirus G2 vaccine for OLP patients and showed the same results in the level of CD4+ and CD8+ T cells as well as CD4+/CD8+ ratio; however, this study did not include any control group. Considering that different agents can lead to OLP, these drugs or rotavirus G2 vaccine might merely increase lymphocytes counts, but might improve the disease symptoms by other mechanisms. Moreover, these drugs may have the placebo effect and decrease pain, pickling, and other subjective symptoms in patients. The results of current study may support this hypothesis that instead of a systemic immunologic disorder, only a local immune mechanism named skin-associated lymphoid tissue (SALT) might be involved in OLP. We recommend the assessing the levels of different subpopulations of lymphocytes by considering various clinical forms of OLP, duration of involve- ment, and intensity of symptoms with more patients and healthy individuals in the future studies.

\section{Acknowledgements}

The authors would like to thanks Dr. Simin Hosseini and staff of Reference laboratory of Rasht City.

\section{Authors' Contributions}

Seyed Javad Kia participated in the design of the study and coordination and statistical analysis; Arash Mansourian participated in reviewing and editing and corresponding the manuscript; Masoumeh Nikkhah performed two-color flow cytometry, approved study design; Nariman Nikparto revised the manuscript draft, corrected the manuscript in final draft; all authors read and approved the final manuscript.

\section{Funding/Support}

This study was funded and supported by the Office of Research of Guilan University of Medical Sciences.

\section{References}

1. Lavanya N, Jayanthi P, Rao UK, Ranganathan K. Oral lichen planus: An update on pathogenesis and treatment. J Oral Maxillofac Pathol.2011;15(2):127-32.

2. Usatine RP, Tinitigan M. Diagnosis and treatment of lichen planus. Am Fam Physician. 2011;84(1):53-60.

3. Scully C, Beyli M, Ferreiro MC, Ficarra G, Gill Y, Griffiths M, et al. Update on oral lichen planus: etiopathogenesis and management. Crit Rev Oral Biol Med. 1998;9(1):86-122.

4. Regezi J, Sciubba J. Oral pathology. 3rd ed. Philadelphia: WB Saunders; 1999.

5. Lodi G, Scully C, Carrozzo M, Griffiths M, Sugerman PB, Thongprasom K. Current controversies in oral lichen planus: report of an international consensus meeting. Part 2. Clinical management and malignant transformation. Oral Surg Oral Med Oral Pathol Oral Radiol Endod. 2005;100(2):164-78.

6. Greenberg G. Red \& white lesions of oral mucosa; Burket's Oral Medicine diagnosis \& treatment. 11 th ed: Hamilton: bc Decker Inc; 2008.

7. Yamamoto T, Yoneda K, Ueta E, Osaki T. Cellular immunosuppression in oral lichen planus. J Oral Pathol Med.1990;19(10):464-70.

8. Carrozzo M, Broccoletti R, Carbone M, Gandolfo S, Garzino P, Cascio G. Phenotypic analysis of peripheral blood cell immunity in Italian patients with different varieties of oral lichen planus. Bull Group Int Rech Sci Stomatol Odontol.1996;39(1-2):33-8.

9. Charazinska-Carewicz K, Ganowicz E, Krol M, Gorska R. Assessment of the peripheral immunocompetent cells in patients with reticular and atrophic-erosive lichen planus. Oral Surg Oral Med Oral Pathol Oral Radiol Endod. 2008;105(2):202-5.

10. Lin SC, Hahn LJ, Kwan HW. Subsets of T lymphocytes in periph eral blood of patients with oral lichen planus. Int J Oral Maxillofac Surg. 1988;17(2):84-6.

11. Walton LJ, Macey MG, Thornhill MH, Farthing PM. Intra-epithelial subpopulations of T lymphocytes and Langerhans cells in oral lichen planus. J Oral Pathol Med.1998;27(3):116-23.

12. Krasowska D, Pietrzak A, Surdacka A, Tuszynska-Bogucka V, Janowski K, Rolinski J. Psychological stress, endocrine and immune response in patients with lichen planus. Int J Dermatol. 2008;47(11):1126-34.

13. al-Fouzan AS, Habib MA, Sallam TH, el-Samahy MH, Rostom AI. Detection of $\mathrm{T}$ lymphocytes and T lymphocyte subsets in lichen planus: in situ and in peripheral blood. Int J Dermatol. 1996;35(6):426-9.

14. Eversole LR. Immunopathogenesis of oral lichen planus 
and recurrent aphthous stomatitis. Semin Cutan Med Surg. 1997;16(4):284-94.

15. Rodriguez-Nunez I, Blanco-Carrion A, Garcia AG, Rey JG. Peripheral T-cell subsets in patients with reticular and atrophic-erosive oral lichen planus. Oral Surg Oral Med Oral Pathol Oral Radiol Endod. 2001;91(2):180-8.

16. Gadenne AS, Strucke R, Dunn D, Wagner M, Bleicher P, Bigby M. T-cell lines derived from lesional skin of lichen planus patients contain a distinctive population of T-cell receptor gamma deltabearing cells. J Invest Dermatol. 1994;103(3):347-51.

17. Pang J, Bu J. [Immunoregulation effect of traditional Chinese medicine treatment on patients with oral lichen planus]. Zhonghua Kou Qiang Yi Xue Za Zhi.1998;33(1):48-9.

18. Kia SJ, Sahebjamee M, Mohaghegh hazrati S, Mighani G, Tohidast Z. The effects of immunotherapeutic G2 vaccine on treatment of oral lichen planus: A pilot study. J Shiraz Univ. 2011. 